\title{
IONIZATION EQUILIBRIUM AND COMPOSITION OF LI-H-U PLASMA
}

\author{
Maratbek Gabdullin, ${ }^{1}$ Tlekkabul Ramazanov, ${ }^{2}$ Zhuldyz Otarbay, ${ }^{3}$ \\ Meruert Mamatova, ${ }^{4}$ Tomiris Ismagambetova ${ }^{5}$
}

\begin{abstract}
In this paper composition of a non-ideal lithium-hydrogen-uranium plasma was studied using the Saha equation. The results obtained in this paper can be used both in theoretical calculations and in the implementation of various experimental projects related to non-ideal plasma, in particular, for studying the thermodynamic properties of nuclear fuel detected in gaseous plasma form in nuclear jet engines. The degree of ionization of lithium, hydrogen, and uranium atoms was determined as the ratio of the number of free electrons to the total number of nuclei in the plasma. The composition of the ionized plasma was calculated in the framework of the system of the chemical model of plasma. A system of nonlinear Saha equations is obtained, which was solved by numerical methods. The calculations were carried out in the Debye approximation, taking into account the screening effects. As a result, the dependence of the degree of ionization of lithium, hydrogen, and uranium atoms on concentration and temperature at different percentages of these atoms in the substance is presented: $10-85-5 \%, 9-90-1 \%$, respectively.
\end{abstract}

UDC Classification:533.9; DOI: http://dx.doi.org/10.12955/cbup.v5.1093

Keywords: plasma, composition, ionization.

\section{Introduction}

The rocket engine is a jet engine that does not use a medium such as air or water to work. Many types of rocket engines such as chemical, electrical, nuclear and others are being developed and tested. In nuclear missile engines, it seems possible to use the colossal energy that is derived from the decomposition of the nuclear "fuel" to heat the gas. The principle of operation of nuclear rocket engines is based on a nuclear reaction or radioactive decay that occurs when the released energy heats a working medium, which can be either reaction products or some other substance, such as hydrogen. By using nuclear rocket engines, it is possible to obtain specific impulse values that are much higher than those provided by chemical rocket engines.

The working body is passed through a nuclear reactor in which the fission reaction of atomic nuclei (for example, uranium) occurs, and at the same time it heats the working body up. Nuclear missile engines do not need an oxidizing agent and therefore only one liquid can be used. As a working fluid, it is advisable to use substances that allow the engine to develop a large traction force. This condition is most fully satisfied by liquid hydrogen, followed by ammonia, hydrazine and water. The processes that release nuclear energy are divided into radioactive transformations, fission reactions of heavy nuclei, and the synthesis of light nuclei. Radioisotope transformations are realized in so-called isotope energy sources. Specific mass energy (the energy that can be extracted by a substance with a mass of 1 $\mathrm{kg}$ ) of artificial radioactive isotopes is much higher than chemical fuels. Thus, for ${ }^{210} \mathrm{Po}$ it is equal to $5 \times 10^{8} \mathrm{~kJ} / \mathrm{kg}$, while for the most energy-efficient chemical fuel (beryllium with oxygen) this value does not exceed $3 \times 10^{4} \mathrm{~kJ} / \mathrm{kg}$. There are many radioactive isotopes, but uranium is more energy-efficient in nuclear reactors. Thus, the specific mass energy of ${ }^{235} \mathrm{U}$ (the fissile uranium isotope) is equal to $6.75 \times 10^{9} \mathrm{~kJ} / \mathrm{kg}$, that is about an order of magnitude higher than that of the isotope ${ }^{210} \mathrm{Po}$ according to the work done by Lioznov (1999).

The study of the thermodynamic properties of nuclear fuel found in a gaseous plasma form in nuclear jet engines is of great interest. Thus, we wrote a program for calculating various proportions of the complex plasma composition. For example, in this paper the results for ionization equilibrium and the composition of the lithium-hydrogen-uranium plasma are presented.

\section{The composition of partially ionized plasma}

The ionization equilibrium and composition of the lithium-hydrogen-uranium plasma were considered in the chemical model of plasma. From work by Gabdullin (2013) we have the following reactions of ionization and recombination of hydrogen, lithium and uranium:

\footnotetext{
${ }^{1}$ NNLOT, al-Farabi Kazakh National University, gabdullin@physics.kz

2 ITEP, al-Farabi Kazakh National University, ramazan@physics.kz

${ }^{3}$ ITEP, al-Farabi Kazakh National University, otarbay@physics.kz

${ }^{4}$ ITEP, al-Farabi Kazakh National University, mira2604@mail.ru

${ }^{5}$ ITEP, al-Farabi Kazakh National University, miriael@ mail.ru
} 


$$
\begin{aligned}
& H \rightarrow H^{+}+e, L i \rightarrow L i^{1+}+e \\
& L^{1+} \rightarrow L^{2+}+e, L i^{2+} \rightarrow L i^{3+}+e \\
& U \rightarrow U^{1+}+e, U^{1+} \rightarrow U^{2+}+e \\
& U^{2+} \rightarrow U^{3+}+e, U^{3+} \rightarrow U^{4+}+e
\end{aligned}
$$

The relationship between the concentrations of plasma components can be described as follows:

$$
\begin{aligned}
& \alpha_{H}^{+}=\frac{n_{H}^{+}}{n_{t o t}}, \alpha_{L i}^{+}=\frac{n_{L i}^{+}}{n_{t o t}}, \alpha_{L i}^{2+}=\frac{n_{L i}^{2+}}{n_{t o t}} \\
& \alpha_{L i}^{3+}=\frac{n_{L i}^{3+}}{n_{t o t}} \alpha_{U}^{+}=\frac{n_{U}^{+}}{n_{t o t}}, \alpha_{U}^{2+}=\frac{n_{U}^{2+}}{n_{t o t}}, \\
& \alpha_{U}^{3+}=\frac{n_{U}^{3+}}{n_{t o t}}, \alpha_{U}^{4+}=\frac{n_{U}^{4+}}{n_{t o t}}, \\
& \alpha_{H}=\frac{n_{H}}{n_{t o t}}, \alpha_{L i}=\frac{n_{L i}}{n_{t o t}}, \alpha_{U}=\frac{n_{U}}{n_{t o t}} .
\end{aligned}
$$

The system of Saha equations for calculating the Li-H-U plasma composition with the maximum ionization multiplicity:

$$
\begin{aligned}
& \frac{n_{H}}{n_{H}^{+} n_{e}^{\text {free }}}=\frac{g_{0}}{g_{1}} \frac{\lambda^{3}}{2} \exp \left(\frac{I_{1}-\Delta I}{k_{B} T}\right), \frac{n_{L i}}{n_{L i}^{+} n_{e}^{\text {free }}}=\frac{g_{0}}{g_{1}} \frac{\lambda^{3}}{2} \exp \left(\frac{I_{2}-\Delta I}{k_{B} T}\right), \\
& \frac{n_{L i}^{+}}{n_{L i}^{2+} n_{e}^{\text {free }}}=\frac{g_{0}}{g_{1}} \frac{\lambda^{3}}{2} \exp \left(\frac{I_{3}-\Delta I}{k_{B} T}\right), \frac{n_{L i}^{2+}}{n_{L i}^{3+} n_{e}^{\text {free }}}=\frac{g_{0}}{g_{1}} \frac{\lambda^{3}}{2} \exp \left(\frac{I_{4}-\Delta I}{k_{B} T}\right) \\
& \frac{n_{U}}{n_{U}^{+} n_{e}^{\text {free }}}=\frac{g_{0}}{g_{1}} \frac{\lambda^{3}}{2} \exp \left(\frac{I_{5}-\Delta I}{k_{B} T}\right), \frac{n_{U}^{+}}{n_{U}^{2+} n_{e}^{\text {free }}}=\frac{g_{0}}{g_{1}} \frac{\lambda^{3}}{2} \exp \left(\frac{I_{6}-\Delta I}{k_{B} T}\right), \\
& \frac{n_{U}^{2+}}{n_{U}^{3+} n_{e}^{\text {free }}}=\frac{g_{0}}{g_{1}} \frac{\lambda^{3}}{2} \exp \left(\frac{I_{7}-\Delta I}{k_{B} T}\right), \frac{n_{U}^{3+}}{n_{U}^{4+} n_{e}^{\text {free }}}=\frac{g_{0}}{g_{1}} \frac{\lambda^{3}}{2} \exp \left(\frac{I_{8}-\Delta I}{k_{B} T}\right) .
\end{aligned}
$$

In these equations $n_{L i}, n_{H}, n_{U}$ are the concentrations of non-ionized $\mathrm{Li}, \mathrm{H}$ and $\mathrm{U}$ ions, respectively, $n_{L i}^{i}, n_{U}^{i}$ are the concentration of i-times ionized $\mathrm{Li}$ and $\mathrm{U}$ ions, $n_{H}^{1+}$ is the concentration of singly ionized $\mathrm{H}$ ions, $n_{t o t}$ is the concentration of all particles, $n_{e}^{\text {free }}$ is the concentration of free electrons of $\mathrm{Li}, \mathrm{H}$ and $\mathrm{U}, I_{i}$ is the ionization potential of $\mathrm{Li}, \mathrm{H}$, and $\mathrm{U}$ atoms, $\Delta I$ is the Debye correction, $g_{0} / g_{1}$ is the partition function.

The law of conservation of the number of nuclei

$$
n_{\text {tot }}=n_{H}+n_{L i}+n_{U}+n_{H}^{+}+n_{L i}^{+}+n_{U}^{+}+n_{L i}^{2+}+n_{U}^{2+}+n_{L i}^{3+}+n_{U}^{3+}+n_{U}^{4+} \text {. }
$$

The law of conservation of the total charge in the system:

$$
n_{e}^{\text {free }}=n_{H}^{+}+n_{L i}^{+}+n_{U}^{+}+2 n_{L i}^{+}+2 n_{U}^{2+}+3 n_{L i}^{3+}+3 n_{U}^{3+}+4 n_{U}^{4+} \text {. }
$$

The system of equations (3) was solved by numerical methods. As a result, the degree of ionization was obtained as a function of concentration and temperature.

The results of the calculations are shown in figures 1-4. Figure 1 presents the results for the ratio of Li$\mathrm{H}-\mathrm{U}$ equal to $10-85-5 \%$. The results of the calculations for the Li-H-U ratio of 9-90-1\% are shown in Figure 2. 
Figure 1: Dependence of the degree of ionization on temperature at $n=10^{21} \mathrm{~cm}^{-3}$ for $10-85-5 \%$ ratio of Li-HU. Black line - the degree of ionization of singly ionized hydrogen, red line - the degree of ionization of singly ionized lithium, blue line - the degree of ionization of doubly ionized lithium, magenta line - the degree of ionization of triply ionized lithium, olive line - the degree of ionization of singly ionized uranium, navy line - the degree of ionization of doubly ionized uranium, violet line - the degree of ionization of triply ionized uranium, purple line - the degree of ionization of four times ionized uranium.

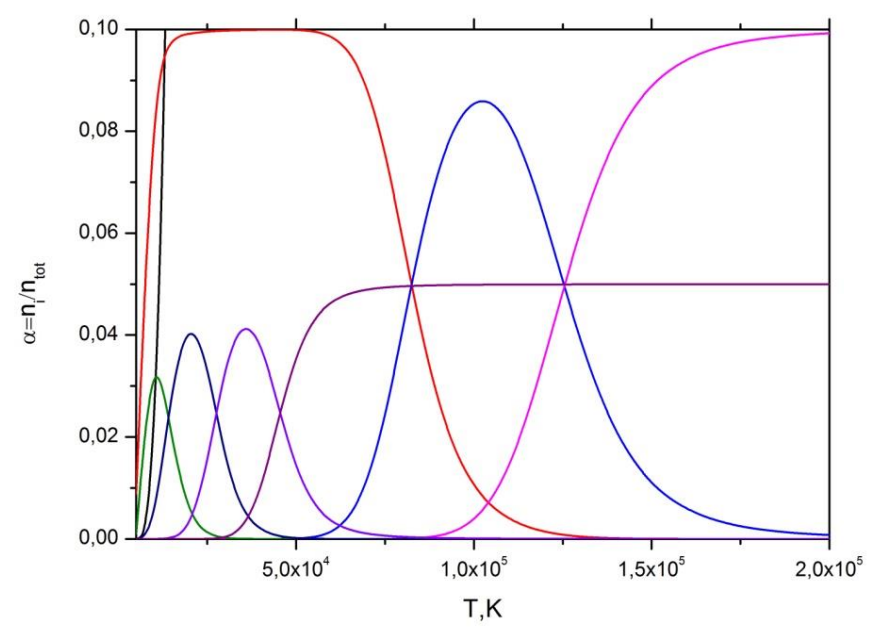

Source: Authors

Figure 2: Dependence of the degree of ionization on temperature at $n=10^{21} \mathrm{~cm}^{-3}$ for 9-90-1\% ratio of Li-HU. Black line - the degree of ionization of singly ionized hydrogen, red line - the degree of ionization of singly ionized lithium, blue line - the degree of ionization of doubly ionized lithium, magenta line - the degree of ionization of triply ionized lithium, olive line - the degree of ionization of singly ionized uranium, navy line - the degree of ionization of doubly ionized uranium, violet line - the degree of ionization of triply ionized uranium, purple line - the degree of ionization of four times ionized uranium.

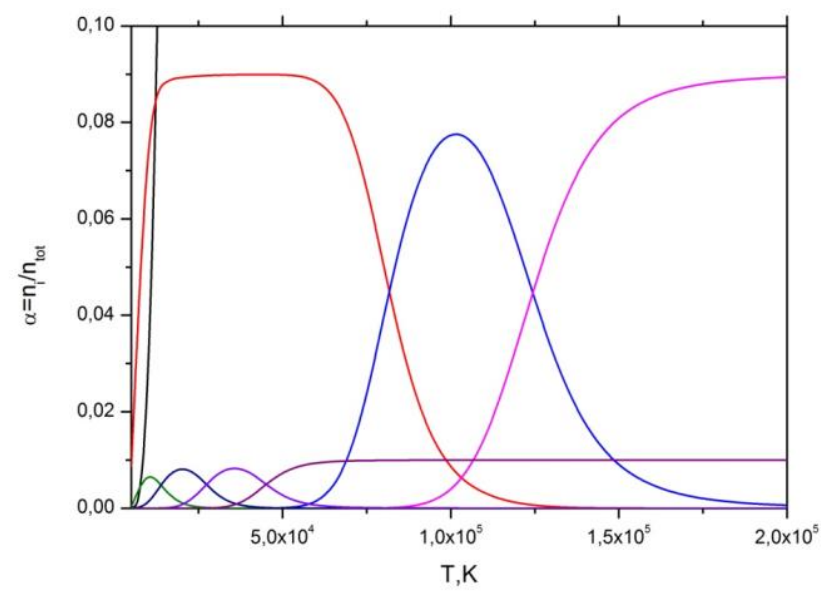

Source: Authors

Figures 1 and 2 show the composition of the Li-H-U plasma as a function of temperature. With increasing temperature the concentration of hydrogen, lithium, and uranium ions increases and, starting from 160,000 K, the plasma becomes completely ionized. It can be seen from the figures that the ionization of hydrogen ions begins at a higher temperature value than for the remaining ions, which is explained by the fact that hydrogen has the highest ionization potential. The concentration of singly ionized lithium increases first, since the ionization potential is the smallest for lithium. The concentration increases with increasing temperature up to $\sim 20,000 \mathrm{~K}$, then reaches a plateau, which is 
due to the need for additional energy to fill the next electron shell. With a further increase in temperature (from $\sim 70000 \mathrm{~K}$ ) the concentration of singly ionized lithium begins to decrease, the concentration of doubly ionized lithium increases, which reaches a maximum at $\sim 110,000 \mathrm{~K}$ and decreases. At the same time, the concentration of triply ionized lithium increases from about $110000 \mathrm{~K}$ to the plateau at $160000 \mathrm{~K}$. The concentration of singly ionized uranium increases with increasing temperature up to $\sim 12000 \mathrm{~K}$, then decreases, the concentration of doubly ionized uranium begins to increase, which reaches a maximum at $\sim 20000 \mathrm{~K}$ and decreases. Simultaneously, the concentration of triply ionized uranium increases and reaches its maximum at $\sim 30000 \mathrm{~K}$ and decreases, the concentration of the four times ionized uranium reaches its plateau at $\sim 70000 \mathrm{~K}$. At $160000 \mathrm{~K}$ the plasma becomes completely ionized.

Figures 3 and 5 show the composition as a function of the concentration at a constant temperature $\mathrm{T}=$ $10000 \mathrm{~K}$. Ionization in dense plasma is a complex process, accompanied by a decrease in the concentration of free electrons and an increase in the concentration of atoms. As the density increases, the average distance between particles decreases, the interaction of the charge with the atomic nucleus increases, and the ionization of atoms is more difficult in dense plasma. The concentration of hydrogen ions gradually decreases with increasing plasma density, the concentration of doubly ionized uranium decreases down to $\mathrm{n}=5 \times 10^{20} \mathrm{~cm}^{-3}$, while the concentration of singly ionized uranium gradually increases.
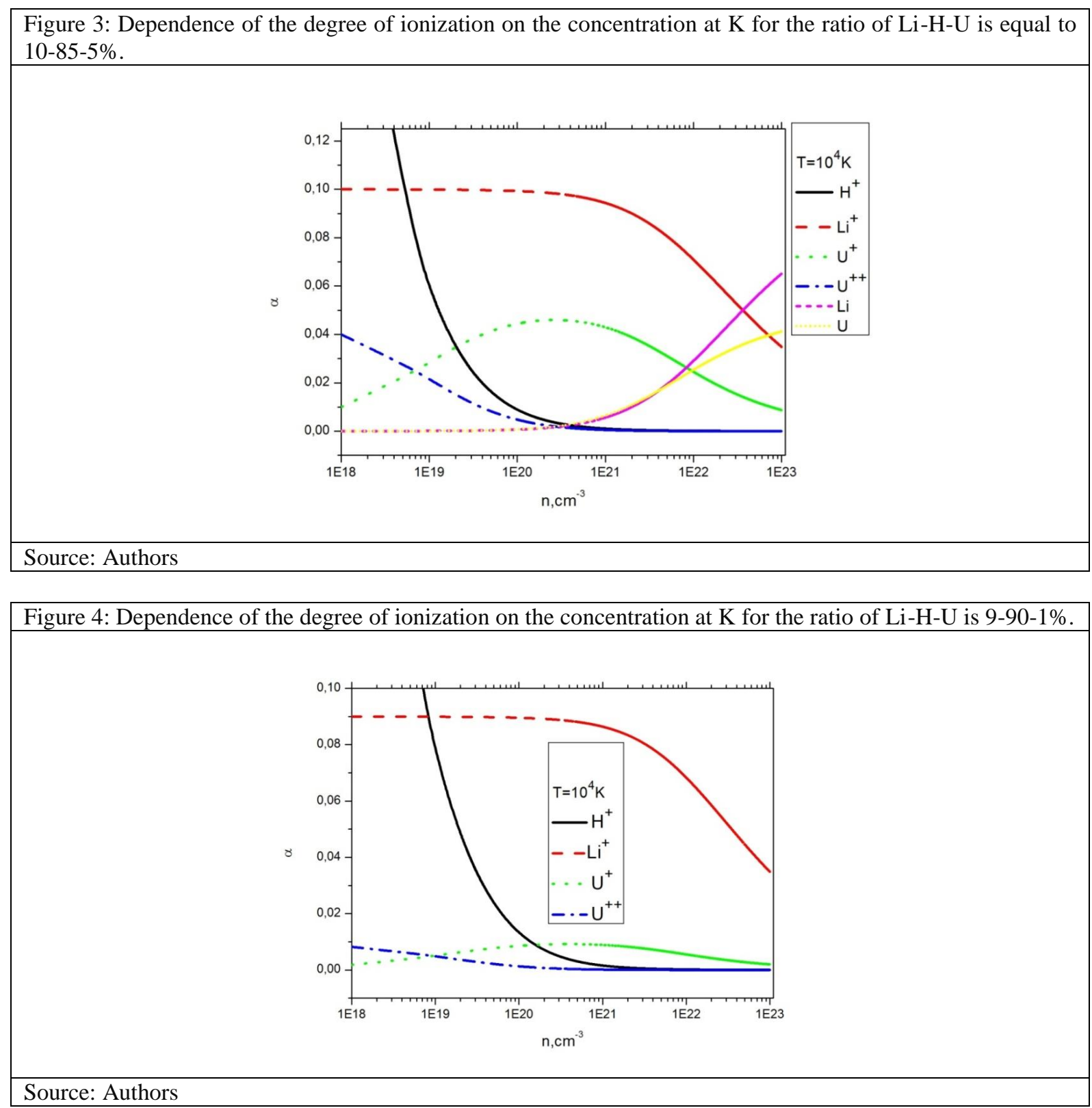


\section{Conclusion}

The composition of non-ideal deuterium and tritium plasma was calculated using the Saha equation while taking into account the lowering of the ionization potential. The composition of dense non-ideal plasma was calculated using numerical methods. The system of Saha nonlinear equations was solved. As a result, the curves showing the degree of ionization depending on concentration and temperature have been obtained.

\section{References}

Gabdullin, M.T., Ramazanov, T.S.,Redmer, R., Akhtanova, G. (2013) Ionization equilibrium and composition of a dense beryllium, boron and carbon plasmas. Contrib. Plasma Phys., 53 (4-5), 311-316.

Lioznov, G. (1999) Gas-phase nuclear engines for space vehicles. Engine, 5 (5), p. 41. 